\title{
On the reciprocal sums of higher-order sequences
}

\section{Zhengang $\mathrm{Wu}^{*}$ and Han Zhang}

"Correspondence: sky.wzgfff@163.com Department of Mathematics, Northwest University, Xi'an, Shaanxi, P.R. China

\section{Abstract}

Let $\left\{u_{n}\right\}$ be a higher-order recursive sequence. Several identities are obtained for the infinite sums and finite sums of the reciprocals of higher-order recursive sequences.

MSC: Primary 11B39

Keywords: infinite sums; finite sums; reciprocal; higher-order recurrences

\section{Introduction}

The so-called Fibonacci zeta function and Lucas zeta function defined by

$$
\zeta_{F}(s)=\sum_{n=1}^{\infty} \frac{1}{F_{n}^{s}} \quad \text { and } \quad \zeta_{L}(s)=\sum_{n=1}^{\infty} \frac{1}{L_{n}^{s}}
$$

where the $F_{n}$ and $L_{n}$ denote the Fibonacci numbers and Lucas numbers, have been considered in several different ways. Navas [1] discussed the analytic continuation of these series. Elsner et al. [2] obtained that for any positive distinct integer $s_{1}, s_{2}, s_{3}$, the numbers $\zeta_{F}\left(2 s_{1}\right), \zeta_{F}\left(2 s_{2}\right)$, and $\zeta_{F}\left(2 s_{3}\right)$ are algebraically independent if and only if at least one of $s_{1}$, $s_{2}, s_{3}$ is even.

Ohtsuka and Nakamura [3] studied the partial infinite sums of reciprocal Fibonacci numbers and proved the following conclusions:

$$
\begin{aligned}
& \left\lfloor\left(\sum_{k=n}^{\infty} \frac{1}{F_{k}}\right)^{-1}\right\rfloor= \begin{cases}F_{n-2} & \text { if } n \text { is even and } n \geq 2 ; \\
F_{n-2}-1 & \text { if } n \text { is odd and } n \geq 1 .\end{cases} \\
& \left\lfloor\left(\sum_{k=n}^{\infty} \frac{1}{F_{k}^{2}}\right)^{-1}\right\rfloor= \begin{cases}F_{n-1} F_{n}-1 & \text { if } n \text { is even and } n \geq 2 ; \\
F_{n-1} F_{n} & \text { if } n \text { is odd and } n \geq 1\end{cases}
\end{aligned}
$$

Where $\lfloor\cdot\rfloor$ denotes the floor function.

Further, Wu and Zhang [4, 5] generalized these identities to the Fibonacci polynomials and Lucas polynomials. Similar properties were also investigated in [6-8]. Related properties of the Fibonacci polynomials and Lucas polynomials can be found in [9-12].

Recently, some authors considered the nearest integer of the sums of reciprocal Fibonacci numbers and other famous sequences and obtained several new interesting identities, see [13] and [14]. Kilic and Arikan [15] defined a $k$ th-order linear recursive sequence

(c) 2013 Wu and Zhang: licensee Springer. This is an Open Access article distributed under the terms of the Creative Commons Attribution License (http://creativecommons.org/licenses/by/2.0), which permits unrestricted use, distribution, and reproduction in any medium, provided the original work is properly cited. 
$\left\{u_{n}\right\}$ for any positive integer $p \geq q$ and $n>k$ as follows:

$$
u_{n}=p u_{n-1}+q u_{n-2}+u_{n-3}+\cdots+u_{n-k},
$$

and they proved that there exists a positive integer $n_{0}$ such that

$$
\left\|\left(\sum_{k=n}^{\infty} \frac{1}{u_{k}}\right)^{-1}\right\|=u_{n}-u_{n-1} \quad\left(n \geq n_{0}\right),
$$

where $\|\cdot\|$ denotes the nearest integer. (Clearly, $\|x\|=\left\lfloor x+\frac{1}{2}\right\rfloor$.)

In this paper, we unify the above results by proving some theorems that include all the results, [3-8] and [13-15], as special cases. We consider the following type of higher-order recurrence sequences. For any positive integer $a_{1}, a_{2}, \ldots, a_{m}$, we define $m$ th-order linear recursive sequences $\left\{u_{n}\right\}$ for $n>m$ as follows:

$$
u_{n}=a_{1} u_{n-1}+a_{2} u_{n-2}+\cdots+a_{m-1} u_{n-m+1}+a_{m} u_{n-m},
$$

with initial values $u_{i} \in \mathbb{N}$ for $0 \leq i<m$ and at least one of them is not zero. If $m=2$, $a_{1}=a_{2}=1$, then $u_{n}=F_{n}$ are the Fibonacci numbers. If $m=2, a_{1}=2, a_{2}=1$, then $u_{n}=P_{n}$ are the Pell numbers. Our main results are the following.

Theorem 1 Let $\left\{u_{n}\right\}$ be an mth-order sequence defined by (1) with the restriction $a_{1} \geq a_{2} \geq$ $\cdots \geq a_{m} \geq 1$. For any positive real number $\beta>2$, there exists a positive integer $n_{1}$ such that

$$
\left\|\left(\sum_{k=n}^{\lfloor\beta n\rfloor} \frac{1}{u_{k}}\right)^{-1}\right\|=u_{n}-u_{n-1} \quad\left(n \geq n_{1}\right) .
$$

Taking $\beta \rightarrow+\infty$, from Theorem 1 we may immediately deduce the following.

Corollary 1 Let $\left\{u_{n}\right\}$ be an mth-order sequence defined by (1) with the restriction $a_{1} \geq a_{2} \geq$ $\cdots \geq a_{m} \geq 1$. Then there exists a positive integer $n_{2}$ such that

$$
\left\|\left(\sum_{k=n}^{\infty} \frac{1}{u_{k}}\right)^{-1}\right\|=u_{n}-u_{n-1} \quad\left(n \geq n_{2}\right) .
$$

For a positive real number $1<\beta \leq 2$, whether there exits an identity for

$$
\left\|\left(\sum_{k=n}^{\lfloor\beta n\rfloor} \frac{1}{u_{k}}\right)^{-1}\right\|
$$

is an interesting open problem.

\section{Several lemmas}

To complete the proof of our theorem, we need the following. 
Lemma 1 Let $a_{1}, a_{2}, \ldots, a_{m}$ be positive integers with $a_{1} \geq a_{2} \geq \cdots \geq a_{m} \geq 1$ and $m \in \mathbb{N}$ with $m \geq 2$. Then, for the polynomial

$$
f(x)=x^{m}-a_{1} x^{m-1}-a_{2} x^{m-2}-\cdots-a_{m-1} x-a_{m},
$$

we have

(I) Polynomial $f(x)$ has exactly one positive real zero $\alpha$ with $a_{1}<\alpha<a_{1}+1$.

(II) Other $m-1$ zeros of $f(x)$ lie within the unit circle in the complex plane.

Proof For any positive integer $a_{1} \geq a_{2} \geq \cdots \geq a_{m} \geq 1$ and $m \geq 2$, we have

$$
\begin{aligned}
f\left(a_{1}\right) & =a_{1}^{m}-a_{1}^{m}-a_{2} a_{1}^{m-2}-\cdots-a_{m-1} a_{1}-a_{m} \\
& =-a_{2} a_{1}^{m-2}-\cdots-a_{m-1} a_{1}-a_{m}<0,
\end{aligned}
$$

and

$$
\begin{aligned}
f\left(a_{1}+1\right) & =\left(a_{1}+1\right)^{m}-a_{1}\left(a_{1}+1\right)^{m-1}-a_{2}\left(a_{1}+1\right)^{m-2}-\cdots-a_{m} \\
& >\left(a_{1}+1\right)^{m}-a_{1}\left(\left(a_{1}+1\right)^{m-1}+\left(a_{1}+1\right)^{m-2}+\cdots+1\right) \\
& =\left(a_{1}+1\right)^{m}-a_{1} \cdot \frac{\left(a_{1}+1\right)^{m}-1}{a_{1}}=1>0 .
\end{aligned}
$$

Thus there exits a positive real zero $\alpha$ of $f(x)$ with $a_{1}<\alpha<a_{1}+1$. According to Descarte's rule of signs, $f(x)=0$ has at most one positive real root. So, $f(x)$ has exactly one positive real zero $\alpha$ with $a_{1}<\alpha<a_{1}+1$. This completes the proof of (I) in Lemma 1 .

Observe that from (I) in Lemma 1 we have

if $x \in \mathbb{R}$ such that $x>\alpha$, then $f(x)>0$,

if $x \in \mathbb{R}$ such that $0<x<\alpha$, then $f(x)<0$.

Let

$$
\begin{aligned}
g(x) & =(x-1) f(x) \\
& =x^{m+1}-\left(a_{1}+1\right) x^{m}+\left(a_{1}-a_{2}\right) x^{m-1}+\left(a_{2}-a_{3}\right) x^{m-2}+\cdots+\left(a_{m-1}-a_{m}\right) x+a_{m} .
\end{aligned}
$$

Since $f(x)$ has exactly one positive real zero $\alpha, g(x)$ has two positive real zeros $\alpha$ and 1 . Observe that

$$
\begin{aligned}
& \text { if } x \in \mathbb{R} \text { such that } x>\alpha \text {, then } g(x)>0, \\
& \text { if } x \in \mathbb{R} \text { such that } 1<x<\alpha \text {, then } g(x)<0 .
\end{aligned}
$$

To complete the proof of (II) in Lemma 1, it is sufficient to show that there is no zero on and outside of the unit circle.

Claim $1 f(x)$ has no complex zero $z_{1}$ with $\left|z_{1}\right|>\alpha$. 
Proof Assume that there exits such a zero. So, we have

$$
f\left(z_{1}\right)=z_{1}^{m}-a_{1} z_{1}^{m-1}-a_{2} z_{1}^{m-2}-\cdots-a_{m-1} z_{1}-a_{m}=0
$$

then we obtain

$$
\begin{aligned}
& \left|z_{1}^{m}\right| \leq a_{1}\left|z_{1}^{m-1}\right|+a_{2}\left|z_{1}^{m-2}\right|+\cdots+a_{m-1}\left|z_{1}\right|+a_{m} \\
& f\left(\left|z_{1}\right|\right)=\left|z_{1}^{m}\right|-a_{1}\left|z_{1}^{m-1}\right|-a_{2}\left|z_{1}^{m-2}\right|-\cdots-a_{m-1}\left|z_{1}\right|-a_{m} \leq 0 .
\end{aligned}
$$

This contradicts with (2).

Claim $2 f(x)$ has no complex zero $z_{2}$ with $1<\left|z_{2}\right|<\alpha$.

Proof Assume that there exits such a zero. Since $f\left(z_{2}\right)=0$,

$$
g\left(z_{2}\right)=z_{2}^{m+1}-\left(a_{1}+1\right) z_{2}^{m}+\left(a_{1}-a_{2}\right) z_{2}^{m-1}+\cdots+\left(a_{m-1}-a_{m}\right) z_{2}+a_{m}=0,
$$

then we obtain

$$
\left(a_{1}+1\right)\left|z_{2}\right|^{m} \leq\left|z_{2}\right|^{m+1}+\left(a_{1}-a_{2}\right)\left|z_{2}\right|^{m-1}+\cdots+\left(a_{m-1}-a_{m}\right)\left|z_{2}\right|+a_{m} .
$$

So, we have $g\left(\left|z_{2}\right|\right) \geq 0$, which contradicts with (5).

Claim 3 On the circle $\left|z_{3}\right|=\alpha$ and $\left|z_{3}\right|=1, f(x)$ has the unique zero $\alpha$.

Proof If $f\left(z_{3}\right)=0$, then

$$
g\left(z_{3}\right)=z_{3}^{m+1}-\left(a_{1}+1\right) z_{3}^{m}+\left(a_{1}-a_{2}\right) z_{3}^{m-1}+\cdots+\left(a_{m-1}-a_{m}\right) z_{3}+a_{m}=0,
$$

then we obtain

$$
\left(a_{1}+1\right)\left|z_{3}\right|^{m} \leq\left|z_{3}\right|^{m+1}+\left(a_{1}-a_{2}\right)\left|z_{3}\right|^{m-1}+\cdots+\left(a_{m-1}-a_{m}\right)\left|z_{3}\right|+a_{m} .
$$

If $z_{3}=\alpha$ or $z_{3}=1$, then $g\left(z_{3}\right)=0$, so (6) must be an equality. Therefore, $z_{3}^{m+1},\left(a_{1}-\right.$ $\left.a_{2}\right) z_{3}^{m-1},\left(a_{2}-a_{3}\right) z_{3}^{m-2}, \ldots,\left(a_{m-1}-a_{m}\right) z_{3}$ and $a_{m}$ all lie on the same ray issuing from the origin. Since $\left(a_{1}-a_{2}\right),\left(a_{2}-a_{3}\right), \ldots, a_{m}$, are all the elements of $\mathbb{R}^{+}, z_{3}^{m+1}, z_{3}^{m-1}, z_{3}^{m-2}, \ldots, z_{3}$ must be the elements of $\mathbb{R}^{+}$. Therefore we obtain $f\left(z_{3}\right) \in \mathbb{R}^{+}$. On the circle $\left|z_{3}\right|=\alpha$ and $\left|z_{3}\right|=1$, there are two conditions $z_{3}=1$ or $z_{3}=\alpha$. Since $f(1) \neq 0, \alpha$ is the unique zero of $f(x)$, Claim 3 holds.

From the three claims, (II) in Lemma 1 is proven.

Lemma 2 Let $m \geq 2$ and let $\left\{u_{n}\right\}_{n \geq 0}$ be an integer sequence satisfying the recurrence formula (1). Then the closed formula of $u_{n}$ is given by

$$
u_{n}=c \alpha^{n}+\mathcal{O}\left(d^{-n}\right) \quad(n \rightarrow \infty)
$$

where $c>0, d>1$, and $a_{1}<\alpha<a_{1}+1$ is the positive real zero off $(x)$. 
Proof Let $\alpha, \alpha_{1}, \ldots, \alpha_{t}$ be the distinct roots of $f(x)=0$, where $f(x)=0$ is the characteristic equation of the recurrence formula (1). From Lemma 1 we know that $\alpha$ is the simple root of $f(x)=0$, then let $r_{j}$, for $j=1,2, \ldots, t$, denote the multiplicity of the root $\alpha_{j}$. From the properties of $m$ th-order linear recursive sequences, $u_{n}$ can be expressed as follows:

$$
u_{n}=c \alpha^{n}+\sum_{i=1}^{t} P_{i}(n) \alpha_{i}^{n}
$$

where

$$
P_{i}(n) \in \mathbb{R}[n], \quad \operatorname{deg} P_{i}(n)=r_{i}-1, r_{1}+r_{2}+\cdots+r_{t}=m-1, \quad \text { and } \quad c \in \mathbb{R} .
$$

For example, for positive integers $1 \leq u, v, w \leq t$, if $\alpha_{u}$ is the simple $\operatorname{root}$ of $f(x)$, then $P_{u}(n)=$ $g_{1}$, where $g_{1} \in \mathbb{R}$, and $\operatorname{deg} P_{u}(n)=0$; if $\alpha_{v}$ is the double root of $f(x)$, then $P_{v}(n)=g_{2} n+g_{3}$, where $g_{2}, g_{3} \in \mathbb{R}$, and $\operatorname{deg} P_{v}(n)=1$; if $\alpha_{w}$ is the multiple root of $f(x)$ with the multiplicity $r_{w}$, then $P_{w}(n)=b_{1} n^{r_{w}-1}+b_{2} n^{r_{w}-2}+\cdots+b_{r_{w}-1} n+b_{r_{w}}$, where $b_{1}, b_{2}, \ldots, b_{r_{w}} \in \mathbb{R}$, and $\operatorname{deg} P_{w}(n)=$ $r_{w}-1$.

From Lemma 1 we have $\left|\alpha_{i}\right|<1$ for $1 \leq i \leq t$. Since each term of tail in (7) goes to 0 as $n \rightarrow \infty$, we can find the constant $M \in \mathbb{R}$ and $d \in \mathbb{R}$ with $d>1$ for $n>n_{0}$ such that

$$
\left|\sum_{i=1}^{t} P_{i}(n) \alpha_{i}^{n}\right| \leq \sum_{i=1}^{t}\left|P_{i}(n) \alpha_{i}^{n}\right| \leq M d^{-n},
$$

which completes the proof (note that if all the roots of $f(x)$ are distinct, we can choose $d^{-1}=\max \left\{\left|\alpha_{1}\right|,\left|\alpha_{2}\right|, \ldots,\left|\alpha_{m-1}\right|\right\}$ and $\left.M=m-1\right)$.

\section{Proof of Theorem 1}

In this section, we shall complete the proof of Theorem 1. From the geometric series as $\epsilon \rightarrow 0$, we have

$$
\frac{1}{1 \pm \epsilon}=1 \mp \epsilon+\mathcal{O}\left(\epsilon^{2}\right)=1+\mathcal{O}(\epsilon)
$$

Using Lemma 2, we have

$$
\begin{aligned}
\frac{1}{u_{k}} & =\frac{1}{c \alpha^{k}+\mathcal{O}\left(d^{-k}\right)}=\frac{1}{c \alpha^{k}\left(1+\mathcal{O}\left((\alpha d)^{-k}\right)\right)} \\
& =\frac{1}{c \alpha^{k}}\left(1+\mathcal{O}\left((\alpha d)^{-k}\right)\right) \\
& =\frac{1}{c \alpha^{k}}+\mathcal{O}\left(\left(\alpha^{2} d\right)^{-k}\right)
\end{aligned}
$$

Thus

$$
\begin{aligned}
\sum_{k=n}^{\lfloor\beta n\rfloor} \frac{1}{u_{k}} & =\frac{1}{c} \sum_{k=n}^{\lfloor\beta n\rfloor} \frac{1}{\alpha^{k}}+\mathcal{O}\left(\sum_{k=n}^{\lfloor\beta n\rfloor}\left(\alpha^{2} d\right)^{-k}\right) \\
& =\frac{\alpha}{c(\alpha-1)} \cdot \alpha^{-n}-\frac{1}{c(\alpha-1)} \cdot \alpha^{-\lfloor\beta n\rfloor}+\mathcal{O}\left(\alpha^{-2 n} d^{-n}\right)
\end{aligned}
$$




$$
\begin{aligned}
& =\frac{\alpha}{c(\alpha-1)} \cdot \alpha^{-n}+\mathcal{O}\left(\alpha^{-2 n} \alpha^{-\lfloor\beta n\rfloor+2 n}\right)+\mathcal{O}\left(\alpha^{-2 n} d^{-n}\right) \\
& =\frac{\alpha}{c(\alpha-1)} \alpha^{-n}+\mathcal{O}\left(\alpha^{-2 n} h\right),
\end{aligned}
$$

where $h=\max \left\{\alpha^{-\lfloor\beta n\rfloor+2 n}, d^{-n}\right\}$.

Taking reciprocal, we get

$$
\begin{aligned}
\left(\sum_{k=n}^{\lfloor\beta n\rfloor} \frac{1}{u_{k}}\right)^{-1} & =\frac{1}{\frac{\alpha}{c(\alpha-1)} \alpha^{-n}\left(1+\mathcal{O}\left(\alpha^{-n} h\right)\right)} \\
& =\frac{\alpha-1}{\alpha} c \alpha^{n}\left(1+\mathcal{O}\left(\alpha^{-n} h\right)\right) \\
& =\frac{\alpha-1}{\alpha} c \alpha^{n}+\mathcal{O}(h) \\
& =u_{n}-u_{n-1}+\mathcal{O}(h) .
\end{aligned}
$$

Since $h=\max \left\{\alpha^{-\lfloor\beta n\rfloor+2 n}, d^{-n}\right\}<1$, there exists $n \geq n_{1}$ sufficient large so that the modulus of the last error term becomes less than $1 / 2$, which completes the proof.

Proof of Corollary 1 From identity (8), we have

$$
\frac{1}{u_{k}}=\frac{1}{c \alpha^{k}}+\mathcal{O}\left(\left(\alpha^{2} d\right)^{-k}\right)
$$

Thus

$$
\sum_{k=n}^{\infty} \frac{1}{u_{k}}=\frac{1}{c} \sum_{k=n}^{\infty} \frac{1}{\alpha^{k}}+\mathcal{O}\left(\sum_{k=n}^{\infty}\left(\alpha^{2} d\right)^{-k}\right)=\frac{\alpha}{c(\alpha-1)} \alpha^{-n}+\mathcal{O}\left(\left(\alpha^{2} d\right)^{-n}\right) .
$$

Taking reciprocal, we get

$$
\begin{aligned}
\left(\sum_{k=n}^{\infty} \frac{1}{u_{k}}\right)^{-1} & =\frac{1}{\frac{\alpha}{c(\alpha-1)} \alpha^{-n}\left(1+\mathcal{O}\left((\alpha d)^{-n}\right)\right)} \\
& =\frac{\alpha-1}{\alpha} c \alpha^{n}\left(1+\mathcal{O}\left((\alpha d)^{-n}\right)\right) \\
& =\frac{\alpha-1}{\alpha} c \alpha^{n}+\mathcal{O}\left(d^{-n}\right) \\
& =u_{n}-u_{n-1}+\mathcal{O}\left(d^{-n}\right) .
\end{aligned}
$$

So, there exists $n \geq n_{2}$ sufficiently large so that the modulus of the last error term becomes less than $1 / 2$, which completes the proof.

\section{Related results}

The following results are obtained similarly.

Theorem 2 Let $\left\{u_{n}\right\}$ be an mth-order sequence defined by (1) with the restriction $a_{1} \geq a_{2} \geq$ $\cdots \geq a_{m} \geq 1$. Let $p$ and $q$ be positive integers with $0 \leq q<p$. For any real number $\beta>2$, 
there exist positive integers $n_{3}, n_{4}$ and $n_{5}$ depending on $a_{1}, a_{2}, \ldots$, and $a_{m}$ such that
(a) $\left\|\left(\sum_{k=n}^{\lfloor\beta n\rfloor} \frac{(-1)^{k}}{u_{k}}\right)^{-1}\right\|=(-1)^{n}\left(u_{n}+u_{n-1}\right) \quad\left(n \geq n_{3}\right)$,
(b) $\left\|\left(\sum_{k=n}^{\lfloor\beta n\rfloor} \frac{1}{u_{p k+q}}\right)^{-1}\right\|=u_{p n+q}-u_{p n-p+q} \quad\left(n \geq n_{4}\right)$,
(c) $\left\|\left(\sum_{k=n}^{\lfloor\beta n\rfloor} \frac{(-1)^{k}}{u_{p k+q}}\right)^{-1}\right\|=(-1)^{n}\left(u_{p n+q}+u_{p n-p+q}\right) \quad\left(n \geq n_{5}\right)$.

For $\beta \rightarrow+\infty$, we deduce the following identity of infinite sum as a special case of Theorem 2.

Corollary 2 Let $\left\{u_{n}\right\}$ be an mth-order sequence defined by (1) with the restriction $a_{1} \geq$ $a_{2} \geq \cdots \geq a_{m} \geq 1$. Let $p$ and $q$ be positive integers with $0 \leq q<p$. Then there exist positive integers $n_{6}, n_{7}$ and $n_{8}$ depending on $a_{1}, a_{2}, \ldots$, and $a_{m}$ such that
(e) $\left\|\left(\sum_{k=n}^{\infty} \frac{(-1)^{k}}{u_{k}}\right)^{-1}\right\|=(-1)^{n}\left(u_{n}+u_{n-1}\right) \quad\left(n \geq n_{6}\right)$,
(f) $\left\|\left(\sum_{k=n}^{\infty} \frac{1}{u_{p k+q}}\right)^{-1}\right\|=u_{p n+q}-u_{p n-p+q} \quad\left(n \geq n_{7}\right)$,
(g) $\left\|\left(\sum_{k=n}^{\infty} \frac{(-1)^{k}}{u_{p k+q}}\right)^{-1}\right\|=(-1)^{n}\left(u_{p n+q}+u_{p n-p+q}\right) \quad\left(n \geq n_{8}\right)$.

Proof We shall prove only (c) in Theorem 2 and other identities are proved similarly. From Lemma 2 we have

$$
\frac{(-1)^{k}}{u_{p k+q}}=\frac{(-1)^{k}}{c \alpha^{p k+q}+\mathcal{O}\left(d^{-p k-q}\right)}=\frac{(-1)^{k}}{c \alpha^{p k+q}}\left(1+\mathcal{O}\left((\alpha d)^{-p k-q}\right)\right) .
$$

Thus

$$
\begin{aligned}
\sum_{k=n}^{\lfloor\beta n\rfloor} \frac{(-1)^{k}}{u_{p k+q}} & =\frac{(-1)^{n} \alpha^{p}}{c \alpha^{p n+q}\left(\alpha^{p}+1\right)}+\frac{(-1)^{n} \alpha^{p}}{c \alpha^{p\lfloor\beta n\rfloor+q}\left(\alpha^{p}+1\right)}+\mathcal{O}\left(\left(\alpha^{2} d\right)^{-p n-q}\right) \\
& =\frac{(-1)^{n} \alpha^{p}}{c \alpha^{p n+q}\left(\alpha^{p}+1\right)}+\mathcal{O}\left(\alpha^{-p\lfloor\beta n\rfloor-q}\right)+\mathcal{O}\left(\alpha^{-2 p n-2 q} d^{-p n-q}\right) \\
& =\frac{(-1)^{n} \alpha^{p}}{c \alpha^{p n+q}\left(\alpha^{p}+1\right)}+\mathcal{O}\left(\alpha^{-2 p n} \alpha^{-p\lfloor\beta n\rfloor+2 p n}\right)+\mathcal{O}\left(\alpha^{-2 p n} d^{-p n}\right) \\
& =\frac{(-1)^{n} \alpha^{p}}{c \alpha^{p n+q}\left(\alpha^{p}+1\right)}+\mathcal{O}\left(\alpha^{-2 p n} h^{p}\right),
\end{aligned}
$$

where $h=\max \left\{\alpha^{-\lfloor\beta n\rfloor+2 n}, d^{-n}\right\}$. 
Taking reciprocal, we get

$$
\begin{aligned}
\left(\sum_{k=n}^{\lfloor\beta n\rfloor} \frac{(-1)^{k}}{u_{p k+q}}\right)^{-1} & =(-1)^{n}\left(c \alpha^{p n+q}+c \alpha^{p n-p+q}\right)\left(1+\mathcal{O}\left(\alpha^{-p n} h^{p}\right)\right) \\
& =(-1)^{n}\left(u_{p n+q}+u_{p n-p+q}\right)+\mathcal{O}\left(h^{p}\right) .
\end{aligned}
$$

Since $h=\max \left\{\alpha^{-\lfloor\beta n\rfloor+2 n}, d^{-n}\right\}<1$, there exists $n \geq n_{5}$ sufficiently large so that the modulus of the last error term becomes less than $1 / 2$, which completes the proof.

\section{Competing interests}

The authors declare that they have no competing interests.

\section{Authors' contributions}

ZW obtained the theorems and completed the proof. $\mathrm{HZ}$ corrected and improved the final version. Both authors read and approved the final manuscript.

\section{Acknowledgements}

The authors express their gratitude to the referee for very helpful and detailed comments. This work is supported by the N.S.F. $(11071194,11001218)$ of P.R. China and G.I.C.F. (YZZ12062) of NWU.

\section{Received: 22 May 2013 Accepted: 4 June 2013 Published: 27 June 2013}

\section{References}

1. Navas, L: Analytic continuation of the Fibonacci Dirichlet series. Fibonacci Q. 39, 409-418 (2001)

2. Elsner, C, Shimomura, S, Shiokawa, I: Algebraic relations for reciprocal sums of odd sums of Fibonacci numbers. Acta Arith. 148(3), 205-223 (2011)

3. Ohtsuka, H, Nakamura, S: On the sum of reciprocal Fibonacci numbers. Fibonacci Q. 46/47, 153-159 (2008/2009)

4. Wu, Z, Zhang, W: The sums of the reciprocal of Fibonacci polynomials and Lucas polynomials. J. Inequal. Appl. 134, $1-8(2012)$

5. Wu, Z, Zhang, W: Several identities involving Fibonacci polynomials and Lucas polynomials. J. Inequal. Appl. 205, 1-14 (2013)

6. Holliday, S, Komatsu, T: On the sum of reciprocal generalized Fibonacci numbers. Integers 11, 441-455 (2011)

7. Zhang, G: The infinite sum of reciprocal of the Fibonacci numbers. J. Math. Res. Expo. 31, 1030-1034 (2011)

8. Zhang, W, Wang, T: The infinite sum of reciprocal Pell numbers. Appl. Math. Comput. 218, 6164-6167 (2012)

9. Falcón, S, Plaza, A: On the Fibonacci k-numbers. Chaos Solitons Fractals 32, 1615-1624 (2007)

10. $\mathrm{Ma}$, R, Zhang, W: Several identities involving the Fibonacci numbers and Lucas numbers. Fibonacci Q. 45, 164-170 (2007)

11. Wang, T, Zhang, W: Some identities involving Fibonacci, Lucas polynomials and their applications. Bull. Math. Soc. Sci. Math. Roum. 55, 95-103 (2012)

12. Yi, Y, Zhang, W: Some identities involving the Fibonacci polynomials. Fibonacci Q. 40, 314-318 (2002)

13. Komatsu, T: On the nearest integer of the sum of reciprocal Fibonacci numbers. Aportaciones Matematicas Investigacion 20, 171-184 (2011)

14. Komatsu, T, Laohakosol, $\mathrm{V}$ : On the sum of reciprocals of numbers satisfying a recurrence relation of order $\mathrm{s}$. J. Integer Seq. 13, Article ID 10.5.8 (2010)

15. Kilic, E, Arikan, T: More on the infinite sum of reciprocal usual Fibonacci, Pell and higher order recurrences. Appl. Math. Comput. 219, 7783-7788 (2013) 